\title{
The usefulness of whole-exome sequencing in routine clinical practice
}

\author{
Alejandro Iglesias, MD1, Kwame Anyane-Yeboa, MD¹, Julia Wynn, MS², Ashley Wilson, $\mathrm{MS}^{3}$, \\ Megan Truitt Cho, $\mathrm{ScM}^{3}$, Edwin Guzman, $\mathrm{MS}^{3}$, Rebecca Sisson, $\mathrm{MS}^{3}$, Claire Egan, $\mathrm{MS}^{3}$ and \\ Wendy K. Chung, MD, PhD2,4
}

Purpose: Reports of the use of whole-exome sequencing in clinical practice are limited. We report our experience with whole-exome sequencing in 115 patients in a single center and evaluate its feasibility and clinical usefulness in clinical care.

Methods: Whole-exome sequencing was utilized based on the judgment of three clinical geneticists. We describe age, gender, ethnicity, consanguinity, indication for testing, family history, insurance, laboratory results, clinician interpretation of results, and impact on patient care.

Results: Most patients were children (78.9\%). The most common indications for testing were birth defects $(24.3 \%)$ and developmental delay (25.2\%). We identified four new candidate human disease genes and possibly expanded the disease phenotypes associated with five different genes. Establishing a diagnosis led to discontinuation of additional planned testing in all patients, screening for additional manifestations in eight, altered management in fourteen, novel therapy in two, identification of other familial mutation carriers in five, and reproductive planning in six.

Conclusion: Our results show that whole-exome sequencing is feasible, has clinical usefulness, and allows timely medical interventions, informed reproductive choices, and avoidance of additional testing. Our results also suggest phenotype expansion and identification of new candidate disease genes that would have been impossible to diagnose by other targeted testing methods.

Genet Med advance online publication 5 June 2014

Key Words: clinical evaluation; genetic testing; undiagnosed genetic disorders; whole-exome sequencing

\section{INTRODUCTION}

The use of whole-exome sequencing (WES) in the clinical setting has increased significantly in the past 2 years since clinical laboratories started offering it. Many patients with rare disorders now have diagnoses made through WES and had previously spent years on an uninformative diagnostic odyssey enduring costly, time consuming, and sometimes invasive procedures associated with medical risks that are stressful for families and providers and imposing a heavy burden on the health-care system. In the postmortem setting, WES offers the opportunity to obtain maximal genetic information when DNA can be limiting. Often WES is a less expensive option than serial genetic testing for conditions characterized by genetic heterogeneity due to involvement of a large number of genes. For families concerned about the risk of recurrence or considering having additional children, time is often precious and female fertility can be limited. For all these reasons, a comprehensive method of genomic testing such as WES is appealing, but data about its use in the clinical setting are limited.

The available evidence of the use of WES in a clinical setting has largely been an extension of a previous research protocol ${ }^{1}$ or a comparison of traditional diagnostic methods and WES. ${ }^{2}$ Here we report a series of our first 115 patients evaluated by
WES as part of routine clinical care in our clinical genetic practice at a single institution. Our patients were tested before the American College of Medical Genetics and Genomics recommendations on incidental findings were implemented by the laboratories ${ }^{3}$; therefore, our study focuses only on the primary findings. Our experience should assist other clinicians in implementing WES into their clinical practice because we have addressed practical concerns including patient education in pretest counseling, consent, insurance coverage, turnaround time, yield of testing, updates of test results, and impact on clinical care. We have found that WES significantly improves our diagnostic ability; we have addressed many of the practical problems of its clinical implementation and routinely use WES as a primary test in patients' genetic evaluation.

\section{MATERIALS AND METHODS}

We retrospectively reviewed the charts of 115 patients who were clinically evaluated by one of three board-certified clinical geneticists (W.K.C., K.A.-Y., and A.I.) and one of six boardcertified genetic counselors (J.W., A.W., E.G., M.T.C., R.S., and C.E.) at Columbia University Medical Center from October 2011 to July 2013 and for whom WES had been completed in that time period. The study was approved by the Columbia University institutional review board. 
All patients had a genetically undefined disorder at the time of the evaluation. Most patients had previously undergone nondiagnostic genetic evaluations including karyotype, fluorescence in situ hybridization, chromosome microarray, molecular testing for specific genes or groups of related genes, and metabolic testing for inborn errors of metabolism (e.g., quantitative plasma amino acids, plasma acylcarnitines, lactic acid, and urine organic acids). The decision to perform WES was based upon the clinical judgment of the clinical geneticist who evaluated the patient. Pretest genetic counseling was provided by certified genetic counselors and/or a clinical geneticist, and informed consent for WES was obtained during a clinical visit. Educational videos were developed to assist with pre-test counseling and are available at http://www.learninggenetics.org. Four clinical laboratories were used for WES: Ambry Genetics, GeneDx, Baylor College of Medicine, and Columbia University Laboratory of Personalized Genomic Medicine. Testing for inpatients was paid for as part of the inpatient hospitalization. Testing for outpatients was paid partly by insurance and partly by the patient. The choice of clinical laboratory was made based on the out-of-pocket expense to patients by comparing the costs between laboratories based on patients' insurance. The clinical services of the laboratories were assumed to be comparable.

We abstracted age, gender, ethnicity, consanguinity, indication for testing, clinical characteristics, family history, insurance, laboratory results, clinician interpretation of results, and clinical implications of the results from the patient's medical records.

We grouped the indications for testing into the following categories: autism, birth defects, cancer, cardiomyopathy, dermatologic, developmental delay/intellectual disability (ID), dysmorphic features, hearing loss, metabolic disorder, myopathy, neurodegenerative, ophthalmologic disease, seizures, skeletal dysplasia, sudden death, and other (Table 1). In cases with more than one feature (e.g., developmental delay and birth defects), the case was categorized by the most medically impactful feature.

Clinical interpretation of the WES results report was done by the ordering geneticist based on the clinical information available, literature review, and clinical judgment. Judgment about clinical relevance was made by the clinician for all variants reported by the laboratory that were possibly but not definitively related to the clinical phenotype. The clinical implications of the results were determined by the patient's physician in terms of specific changes in management that resulted from a definitive diagnosis and included reproductive planning, identifying additional mutation carriers in the family and additional clinical disease manifestations, and starting or discontinuing specific therapies and/or diagnostic tests.

\section{RESULTS}

The patient population consisted of 59 males (51.3\%) and 56 females (48.6\%), for a total of 115 , of whom $91(78.9 \%)$ were children, 21 (18.2\%) were adults, and $3(2.6 \%)$ were fetal cases from terminated pregnancies (Table 1 ). The ethnic distribution was $61.7 \%$ Caucasian, 20\% Hispanic, 10.4\% Asian, 6\% African American, $0.9 \%$ mixed race, and $0.9 \%$ unknown (adopted).
The most common indications for WES evaluation were birth defects in $24.3 \%$, developmental delay in $25.2 \%$, and seizures in $14 \%$ (Table 1). There was consanguinity in $11.3 \%$ of the cases. Families with more than one affected member accounted for $5.6 \%$ of the 115 patients.

Insurance coverage during the time of the study was obtained from different sources, including 86 patients with private insurance (74.7\%), 8 with Medicaid (6.4\%), 19 with MedicaidHMO (15.2\%), 1 with Medicare (0.8\%), and 1 self-pay (0.8\%) (Table 1). Turnaround time varied among laboratories, but on average, results were received within 4 months, with one laboratory returning results within 6 months.

Out of the 115 cases, a definitive diagnosis was made in 37 cases (32.2\%) (Table 2). In two cases, mutations were identified in SERAC1 and RIT1 after the original report was issued, based on new published information available after the initial analysis. The individual yield for each category was 53.5 $\%$ for birth defects (15/28), 34.4\% for developmental delay/ intellectual disability (10/29), three of seven for cardiomyopathies, three of four for ophthalmologic disease, two of four for myopathies, two of four for dermatologic diseases, two of two for neurological/neurodegenerative disorders, and one of two for metabolic disorder. No diagnoses were established for the categories of autism, hearing loss, seizures, cancer, sudden death, or skeletal dysplasia. Two positive cases were studied at Baylor School of Medicine laboratories; these have been previously reported ${ }^{4}$ and are described in Table 2.

Of the 37 positives cases, 22 were autosomal dominant $(59.45 \%), 13$ were autosomal recessive $(35.15 \%)$, and 2 were X-linked (5.4\%), similar to the distribution of previous reports. ${ }^{4}$ De novo mutations were identified in 15 (40.5\%) cases. Gonadal mosaicism was identified in one case. Two patients were found to have mutations in two different genes and therefore were diagnosed with two conditions.

Four new candidate disease genes were identified, including glutamate pyruvate transaminase 2 (GPT2), myosin heavy chain 10 (MYH10), synaptosomal-associated protein 25 (SNAP25), and transmembrane protein 107 (TMEM107) (Table 3).

Finally, based on WES results, the phenotypes of five clinical conditions were possibly expanded, including those of three patients with mutations in ACTG2 (previously associated with autosomal dominant familial visceral myopathy), one patient with Goldberg-Shprintzen syndrome (KIAA1279), one patient with nemaline myopathy and congenital fiber type disproportion (TPM3), one patient with progressive ophthalmic ophthalmoplegia syndrome (POLG2), and one patient with autosomal dominant mental retardation type 7 (DYRK1A) (Table 2).

\section{DISCUSSION}

We performed WES for clinical diagnostic purposes in 115 patients, identifying a definitive genetic etiology in $32.1 \%$ of our cases. This level of yield is similar to or slightly higher than those of previous reports, either in research studies ${ }^{1,5-7}$ or clinical series reported by clinical laboratories. ${ }^{4}$ 
Table 1 Characteristics of the 115 patients evaluated by clinical whole-exome sequencing

\begin{tabular}{|c|c|}
\hline \multicolumn{2}{|l|}{ Age } \\
\hline Prenatal & $3(2.6 \%)$ \\
\hline Newborn (0-30 days) & $1(0.9 \%)$ \\
\hline Infant (1-12 months) & $7(6 \%)$ \\
\hline Children ( $1-18$ years) & $83(72.1 \%)$ \\
\hline Adult & $21(18.2 \%)$ \\
\hline \multicolumn{2}{|l|}{ Gender } \\
\hline Male & $59(51.3 \%)$ \\
\hline Female & $56(48.6 \%)$ \\
\hline \multicolumn{2}{|l|}{ Ethnicity } \\
\hline Caucasian & $71(61.7 \%)$ \\
\hline Hispanic & $23(20 \%)$ \\
\hline Asian & $12(10.4 \%)$ \\
\hline African American & $7(6 \%)$ \\
\hline Mixed race & $1(0.9 \%)$ \\
\hline Unknown (adopted) & $1(0.9 \%)$ \\
\hline \multicolumn{2}{|l|}{ Consanguinity } \\
\hline Present & $13(11.3 \%)$ \\
\hline Family history of the condition & $7(5.6 \%)$ \\
\hline \multicolumn{2}{|l|}{ Samples submitted } \\
\hline Parent/proband trio & $95(82.6 \%)$ \\
\hline Proband + one parent & $6(5.2 \%)$ \\
\hline Proband only & $3(2.6 \%)$ \\
\hline Proband + other family member $(\mathrm{s})^{\mathrm{a}}$ & $11(9.5 \%)$ \\
\hline \multicolumn{2}{|l|}{ Patient location } \\
\hline Inpatient & $29(25.2 \%)$ \\
\hline Outpatient & $86(74.7 \%)$ \\
\hline \multicolumn{2}{|l|}{ Insurance } \\
\hline Private & $86(74.7 \%)$ \\
\hline Medicaid & $8(6.4 \%)$ \\
\hline Medicaid-HMO & $19(15.2 \%)$ \\
\hline Medicare/out-of-pocket & $1(0.8 \%)$ \\
\hline Self-pay & $1(0.8 \%)$ \\
\hline \multicolumn{2}{|l|}{ Main clinical indication for testing } \\
\hline Autism & $4(3.4 \%)$ \\
\hline Birth defects ${ }^{b}$ & $28(24.3 \%)$ \\
\hline Cancer & $2(1.7 \%)$ \\
\hline Cardiomyopathy & $7(6 \%)$ \\
\hline Dermatologic disease & $4(3.4 \%)$ \\
\hline Developmental delay/intellectual disability & $29(25.2 \%)$ \\
\hline Dysmorphic features & $3(2.6 \%)$ \\
\hline Hearing loss & $2(1.7 \%)$ \\
\hline Metabolic disorder & $2(1.7 \%)$ \\
\hline Myopathy & $4(3.4 \%)$ \\
\hline Neurological/neurodegenerative disorder & $2(1.6 \%)$ \\
\hline Ophthalmologic disease & $4(3.4 \%)$ \\
\hline Seizures & $14(12.1 \%)$ \\
\hline Skeletal dysplasia & $1(0.8 \%)$ \\
\hline Sudden death & $2(1.7 \%)$ \\
\hline Other & $7(6 \%)$ \\
\hline Total & 115 \\
\hline
\end{tabular}

aProband + other family member(s): proband and other family members other than the two parents, often including other affected family members. ${ }^{\text {BBirth }}$ defects: macrocephaly, microcephaly, cleft lip, micrognathia, tetralogy of Fallot, micropenis, hydronephrosis, cryptorchidism, neurogenic bladder, neurogenic bowel, atrial septal defect, agenesis of corpus callosum, abnormal cavum septum pellucidum, mesocephalic clefting, hydrocephalus, Dandy-Walker malformation with aqueductal stenosis, microphthalmia, hypoplastic left heart, microcolon, polydactyly, cystic kidneys, dysplastic kidney, bilateral vertical talus, congenital tongue cysts, and brachial fistulae and cysts. 'Other: gait abnormalities, chronic pain, pleuroparenchimal fibroelastosis, lipodystrophy, connective tissue disorder with severe postural orthostatic tachycardia syndrome, chronic migraines, pain, sinus problems, family history of seizures and microcephaly, and hemolytic anemia.
Our finding of a definitive genetic etiology for developmental delay in $34 \%$ is comparable to those of two research studies on a series of patients with IDs. ${ }^{8,9}$ In one WES study in 100 patients with severe nonsyndromic ID (IQ < 50), De $\mathrm{Ligt}^{8}$ reported a diagnostic yield of $16 \%$. In a second study, Rauch ${ }^{9}$ reported a yield of $45 \%$ in 51 patients with nonsyndromic ID with IQ $<60$. Interestingly, in both studies about $50 \%$ of the patients presented with ID plus at least one additional phenotypic finding (i.e., microcephaly, hypotonia, or seizures). With respect to our series of patients with developmental delay/ID, we found that 7 of 18 patients (34\%) had a definitive diagnosis by WES. When we analyzed our results based on indication, we found that the more narrowly defined the phenotype, the higher the yield. For welldefined phenotypes for which a likelihood of a genetic etiology was high, although our population was extremely small, the yield was between 75 and 100\% (ophthalmologic and specific neurologic indications). Notably, for birth defects, the diagnostic yield was high at 53.5\%. Our results show that even for a wide range of indications, WES has excellent power as a diagnostic tool.

One important diagnostic issue is that young patients may not yet manifest all the signs and symptoms of a given condition, and therefore it may be especially difficult to make a diagnosis early in life, particularly in infancy. Hence a diagnosis made early can be particularly valuable to identify additional associated features of clinical syndromes before they become symptomatic, to prevent or ameliorate the manifestations and to minimize the diagnostic evaluation of new symptoms. This was evident in our patients diagnosed with Coffin-Siris syndrome, Bardet-Biedl syndrome, and Achalasia-AddisonianismAlacrimia syndrome (AAA or Allgrove syndrome).

The field of genetics is rapidly changing, with the ongoing discovery of new genes and syndromes. In this context, two of our patients that did not have a diagnosis based on the original WES results were subsequently found to have one after genes for their diseases were reported in the literature and accordingly the original WES results were revised. One patient with optic atrophy, hearing loss, developmental regression, and 3-methyl glutaconic aciduria was found to be a compound heterozygote for two clear loss-of-function mutations in serine active site containing 1 (SERAC1) (c.438delC (p.T147fs) and c.442C >T (p.R148X)), which has recently been associated with MEGDEL (3-methylglutaconic aciduria with sensorineural deafness and Leigh-like) syndrome. ${ }^{10}$ The second patient had dysmorphic facial features, hypertrophic cardiomyopathy, pulmonic stenosis, and hypotonia suggestive of Noonan syndrome. WES identified a de novo mutation in Ras-like without CAAX 1 (RIT1) (c.170 C $>$ G, p. A57G), which is involved in RAS signaling. A week after the receipt of the clinical report, a paper was published describing mutations in the RIT1 gene as a new cause of Noonan syndrome. ${ }^{11}$ Because the genetic literature is rapidly changing as new genes are identified for human diseases, it is critical that laboratories maintain a database of the variants identified for patients and reassess the significance of these variants over time.

Using WES, we identified new mutations in three patients with megacystis microcolon hypoperistalsis syndrome in the ACTG2 


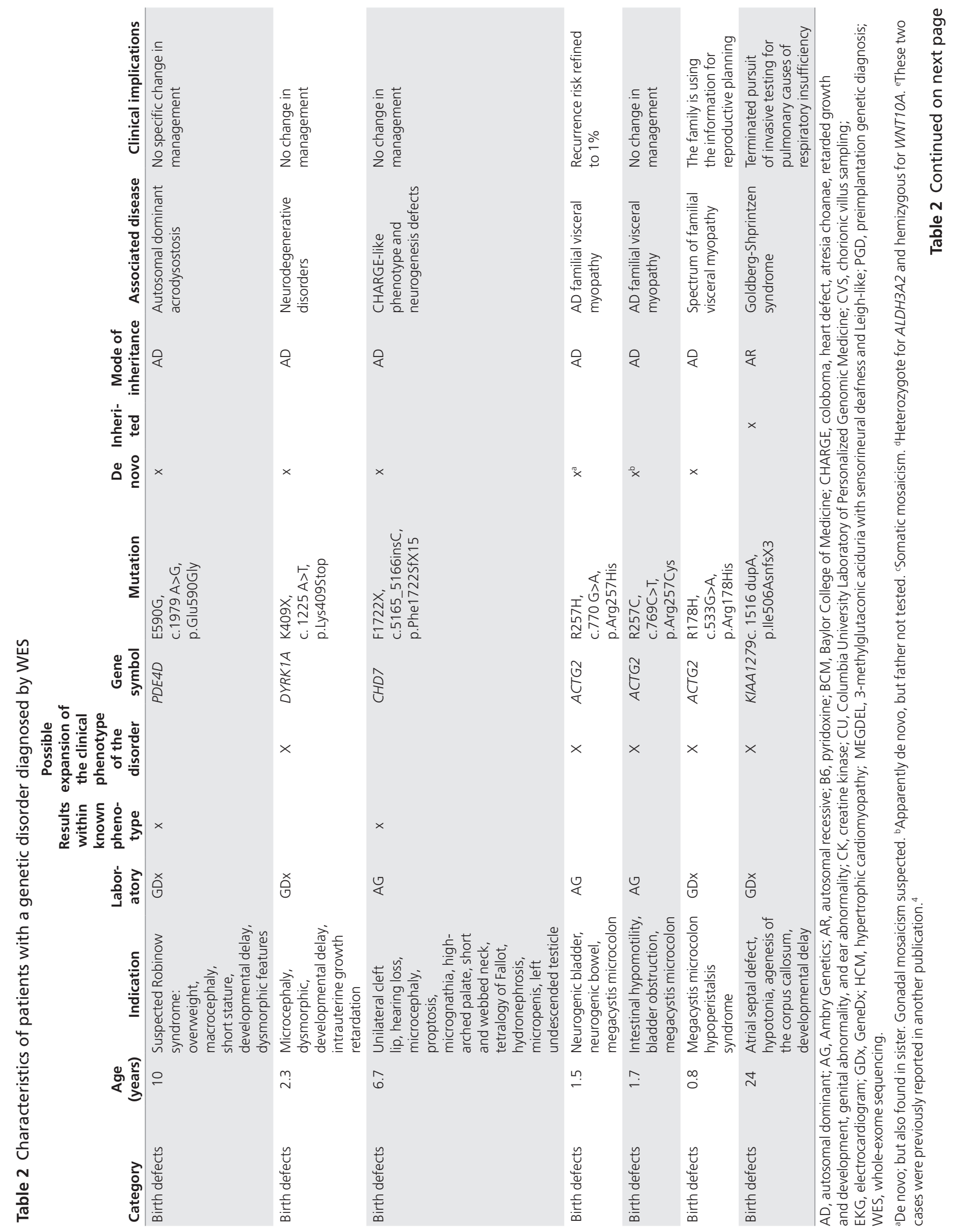




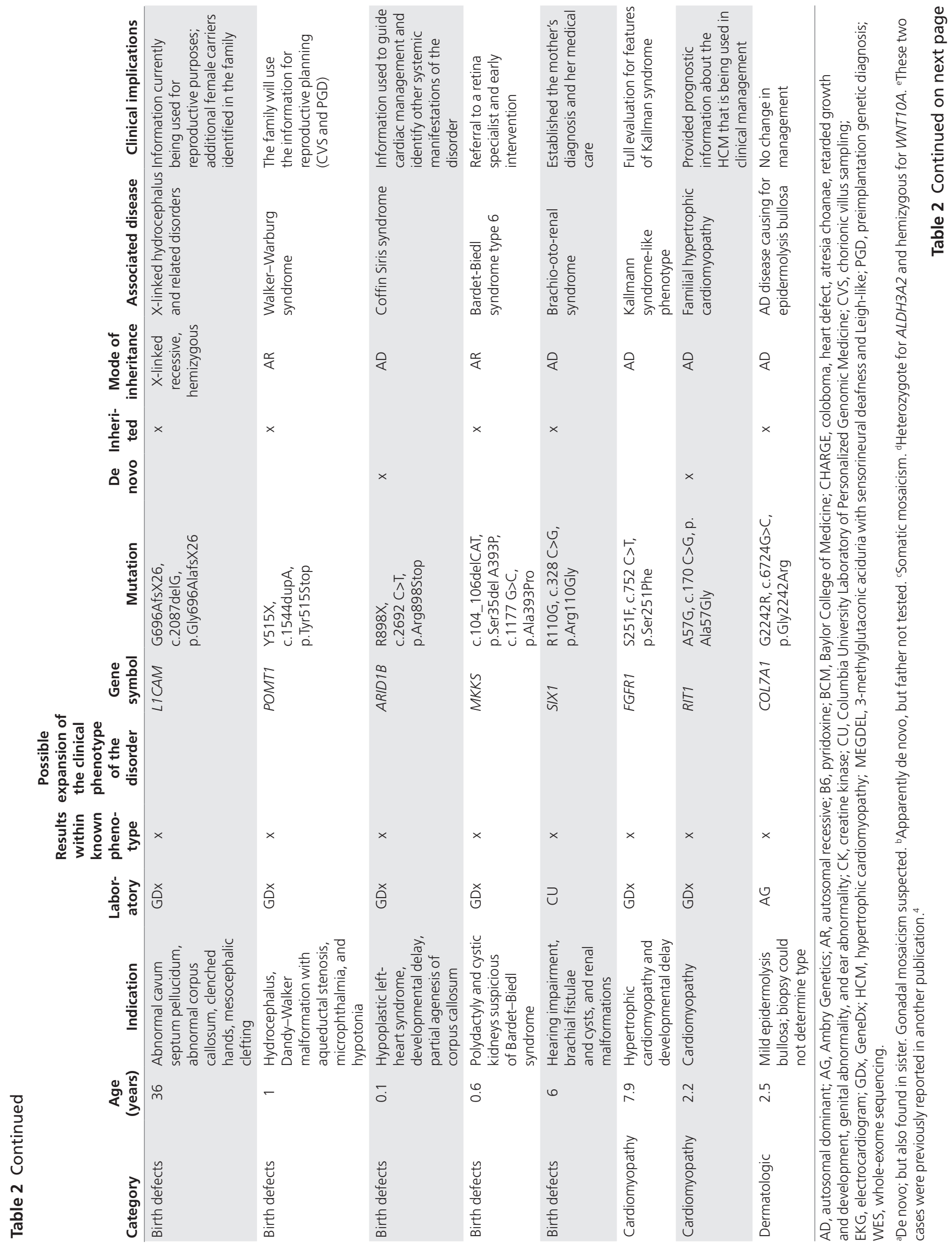




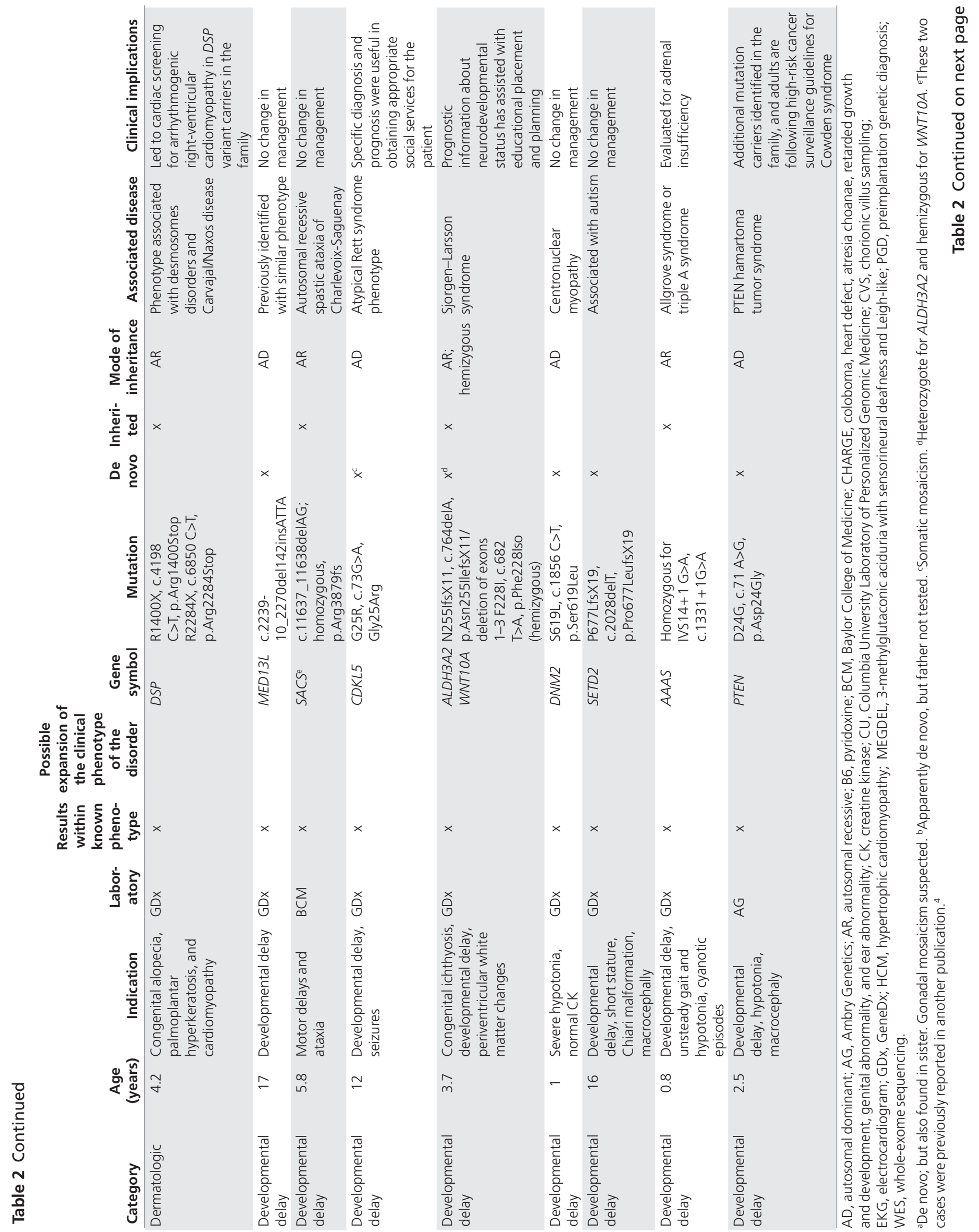




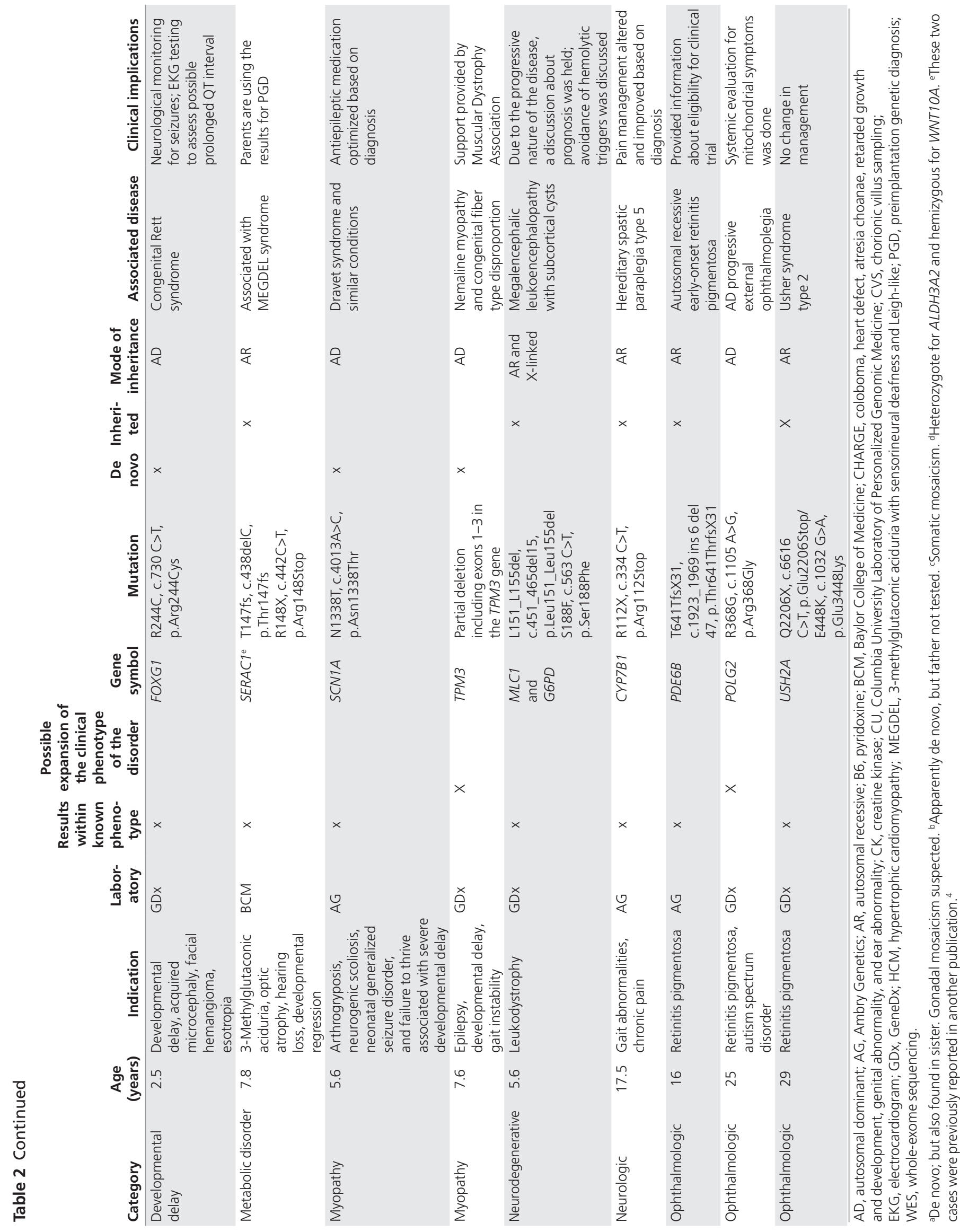




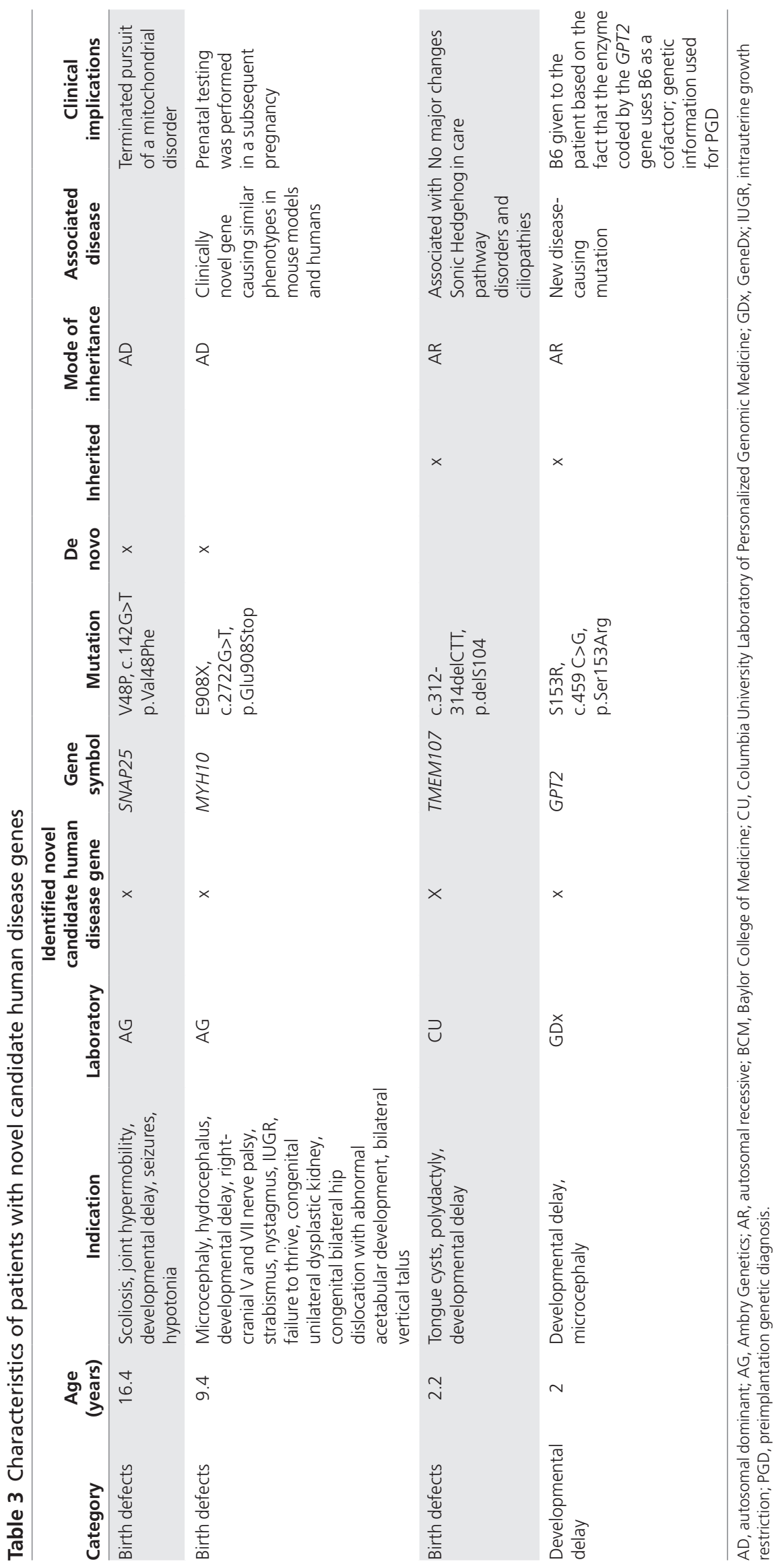


gene, which has been associated with familial visceral myopathy. ${ }^{12}$ In addition, if new patients are identified with features similar to those of our patients with Goldberg-Shprintzen syndrome (KIAA1279), nemaline myopathy and congenital fiber-type disproportion (TPM3), and autosomal dominant mental retardation type 7 (DYRK1A), the features of the conditions should be redefined (Table 2). In the patient with Goldberg-Shprintzen syndrome, one of the cardinal features that originally defined the syndrome, Hirschprung disease, was absent. The patient with nemaline myopathy presented with epilepsy and developmental delay, which are usually not cardinal features of this condition. Finally, the patient with autosomal dominant mental retardation type 7 has a history of intrauterine growth retardation, synophrys, long eyelashes, and anteriorly displaced ears, which have not been previously reported. It is also possible that the patients with nemaline myopathy and autosomal dominant mental retardation type 7 have another etiology for the additional clinical features not previously associated with these conditions.

We identified four new candidate disease genes using clinical WES (Table 3). A de novo mutation in MYH10 that causes a similar phenotype in mouse models; in our patient it caused microcephaly, hydrocephalus, developmental delay, right-cranial V and VII nerve palsy, strabismus, nystagmus, intrauterine growth restriction, failure to thrive, congenital unilateral dysplastic kidney, congenital bilateral hip dislocation with abnormal acetabular development, and bilateral vertical talus. ${ }^{13}$ We also identified a de novo mutation in SNAP25 associated with epilepsy and static encephalopathy. ${ }^{14}$ We identified a homozygous mutation in GPT2 in a patient with developmental delay and microcephaly who has two similarly affected siblings. This gene encodes one of the two pyridoxal enzymes that catalyze a reversible transamination reaction to yield glutamate and pyruvate, and this enzyme participates in amino acid metabolism and neurotransmitter production. Finally, a patient with tongue cysts, polydactyly, and developmental delay, phenotypically overlapping with orofascio-digital syndrome, was homozygous for an in-frame deletion in TMEM107. TMEM107 is a key component of the Sonic Hedgehog pathway and ciliogenesis, which are implicated in normal facial, oral, and acral development. ${ }^{15}$ It is worth noting that the clinical laboratories reported new candidate disease genes in 18 cases. However, upon critical review of the literature, model organisms, and patients' phenotypic features, we considered only four of these to be viable new candidate genes. In this context, clinical comprehensive WES provides a powerful tool for gene discovery and therefore has an advantage over targeted sequencing of subgenomic libraries of all disease genes because $8.1 \%$ of the cases we diagnosed were for novel candidate disease genes not previously known to be associated with human disease.

Besides the obvious implications of providing a patient with a definitive diagnosis of a genetic disorder (i.e., prognostic information, anticipatory care, reproductive risk assessment, and reproductive options), we were able to intervene in specific ways and therefore impact patient care. The use of pyridoxine, vitamin B6, in the three siblings with mutations in the GPT2 gene exemplifies this issue. After the identification of a mutation in the SCN1A gene in a patient with a Dravet-like phenotype, tailored antiepileptic treatment was possible. In several cases, establishing a definitive diagnosis saved the patient from additional invasive testing such as muscle biopsies. In the case of retinitis pigmentosa, the results of genomic testing determined eligibility for clinical trials. Provision of accurate prognostic information, although not medically actionable, was used by the families to obtain the appropriate social services and educational services and was personally actionable as they made personal and financial plans based on the prognosis (Table 2). This issue is extremely important because the provision and coverage of medical, educational, and ancillary services to patients with genetic disorders relies heavily on a confirmed diagnosis to provide prognostic information. In summary, we were able to discontinue further diagnostic investigations in all diagnosed patients, screening for other manifestations in 8, altering management in 14 , changing treatment in 2 , identifying other family member mutation carriers in 5 , and assisting with reproductive planning in 6 families.

Insurance covered WES for the majority of our patients, but this is still an evolving issue. In our series, we were able to obtain coverage with private insurance and Medicaid in 90 and $80 \%$ of patients, respectively. Coverage for Medicaid patients was not provided for the three major commercial laboratories. No Medicare cases were covered. The one Medicare case we studied was covered by out-of-pocket expenses. We also acknowledge that it is not always possible to obtain insurance coverage for all patients and, therefore, WES is not universally accessible to all patients for whom it is medically indicated, although in our opinion it should be.

We spent an average of 30 minutes during the pretest counseling session to consent patients for WES. We are currently using a multimedia presentation explaining WES and secondary findings to more efficiently and consistently provide patient education (http://www.learninggenetics.org). An ongoing assessment of its impact and efficacy is currently in progress.

In conclusion, our results show that WES is an effective tool to diagnose genetically heterogeneous disorders in a clinical setting, especially in young children in whom all the clinical features may not yet be evident. It is also valuable in providing evidence for possible expansion of the phenotypes associated with recognized human diseases and to identify new candidate genes associated with human disease. We were largely successful in obtaining insurance coverage for most or all of the cost of WES, demonstrating the practical feasibility of its use in the clinical setting. Expanded use of WES will improve our ability to diagnose patients with genetic disorders; it will also help us to tailor the medical management of these disorders, anticipate associated medical problems, avoid expensive and sometimes invasive tests, accurately predict the risk of recurrence for parents and other family members, and accurately identify other family members carrying the mutation in order to enable preventative medical care and reproductive planning. Additional studies are necessary to quantify the financial, medical, and emotional benefits of reducing the time and cost of diagnosis. 
Prompt diagnosis has significant implications for families concerned about the risk of recurrence, who are often wary of having additional children before a diagnosis is established. Improvements in sequence coverage, sequence alignment, variant calls, copy-number variant detection, predictions of pathogenicity, increasing number of human disease genes identified, and possibly whole-genome sequencing will only improve the diagnostic yield of genomic testing. Hence, our yield of $32 \%$ should be considered the lower bound of clinical sensitivity for exome sequencing.

\section{ACKNOWLEDGMENT}

We acknowledge the contribution of our patients to this study.

\section{DISCLOSURE}

The authors declare no conflict of interest.

\section{REFERENCES}

1. Jacob HJ, Abrams K, Bick DP, et al. Genomics in clinical practice: lessons from the front lines. Sci Trans/ Med 2013 5:194.

2. Shashi V, McConkie-Rosell A, Rosell B, et al. The utility of the traditional medical genetics diagnostic evaluation in the context of nextgeneration sequencing for undiagnosed genetic disorders. Genet Med 2014;16:176-182.

3. Green RC, Berg JS, Grody WW, et al. ACMG recommendations for reporting of incidental findings in clinical exome and genome sequencing. Genet Med 2013:15:565-574.

4. Yang Y, Muzny DM, Reid JG, et al. Clinical whole-exome sequencing for the diagnosis of mendelian disorders. N Engl J Med 2013;369:1502-1511.
5. Dimmock D. Whole genome sequencing: a considered approach to clinical implementation. Curr Protoc Hum Genet 2013; Chapter 9:Unit 9.22.

6. Yu Y, Wu BL, Wu J, Shen Y. Exome and whole-genome sequencing as clinical tests: a transformative practice in molecular diagnostics. Clin Chem 2012:58:1507-1509.

7. Thompson R, Drew CJ, Thomas RH. Next generation sequencing in the clinical domain: clinical advantages, practical, and ethical challenges. Adv Protein Chem Struct Biol 2012;89:27-63.

8. de Ligt J, Willemsen $\mathrm{MH}$, van Bon BW, et al. Diagnostic exome sequencing in persons with severe intellectual disability. N Eng/ J Med 2012;367:19211929.

9. Rauch A, Wieczorek D, Graf E, et al. Range of genetic mutations associated with severe non-syndromic sporadic intellectual disability: an exome sequencing study. Lancet 2012;380:1674-1682.

10. Sarig O, Goldsher D, Nousbeck J, et al. Infantile mitochondrial hepatopathy is a cardinal feature of MEGDEL syndrome (3-methylglutaconic aciduria type IV with sensorineural deafness, encephalopathy and Leigh-like syndrome) caused by novel mutations in SERAC 1. Am J Med Genet A 2013:161:2204-2215.

11. Aoki Y, Niihori T, Banjo T, et al. Gain-of-function mutations in RIT1 cause Noonan syndrome, a RAS/MAPK pathway syndrome. Am J Hum Genet 2013;93:173-180.

12. Lehtonen HJ, Sipponen T, Tojkander S, et al. Segregation of a missense variant in enteric smooth muscle actin $\gamma$-2 with autosomal dominant familial visceral myopathy. Gastroenterology 2012;143:1482-1491.e3.

13. Tuzovic L, Anyane-Yeboa K, Mills A, Glassberg K, Miller R. Megacystismicrocolon-intestinal hypoperistalsis syndrome: case report and review of prenatal ultrasonographic findings. Fetal Diagn Ther 2014; e-pub ahead of print 21 February 2014

14. Rohena L, Neidich J, Cho T, et al. Mutation in SNAP-25 as a novel genetic cause of epilepsy and intellectual disability. Rare Dis 2013; 1:e26314; doi: 10.4161/ rdis.26314.

15. Christopher KJ, Wang B, Kong Y, Weatherbee SD. Forward genetics uncovers Transmembrane protein 107 as a novel factor required for ciliogenesis and Sonic hedgehog signaling. Dev Bio/ 2012;368:382-392. 